\title{
Prognostic Value of Complement Component 2 and Its Correlation with Immune Infiltrates in Hepatocellular Carcinoma
}

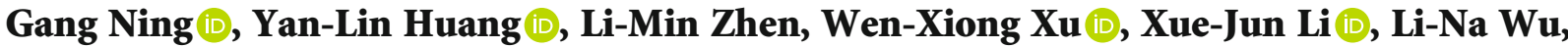 \\ Ying Liu, Chan Xie $\mathbb{i D}^{\text {, } \text {, and Liang Peng }}$
}

Department of Infectious Diseases, The Third Affiliated Hospital of Sun Yat-sen University, Guangzhou, China

Correspondence should be addressed to Chan Xie; happyxiechan@hotmail.com and Liang Peng; pliang@mail.sysu.edu.cn

Received 21 March 2020; Revised 20 May 2020; Accepted 25 May 2020; Published 15 June 2020

Guest Editor: Tao Huang

Copyright (C) 2020 Gang Ning et al. This is an open access article distributed under the Creative Commons Attribution License, which permits unrestricted use, distribution, and reproduction in any medium, provided the original work is properly cited.

\begin{abstract}
Background. Single nucleotide polymorphism (SNP) of complement component 2 (C2) has been found to be significantly associated with hepatocellular carcinoma (HCC). However, little is known about the role and mechanism of C2 in HCC. In the present study, we aimed to explore the prognostic value of C2 and its correlation with tumor-infiltrating immune cells in HCC. Materials and Methods. mRNA expression was downloaded from TCGA (365 HCC patients and 50 healthy controls), GSE14520 (220 HCC patients and 220 adjacent normal tissues), and ICGC HCC (232 HCC patients) cohorts. Unpaired Student's $t$-tests or ANOVA tests were used to evaluate differences of C2 expression. Univariate and multivariate analyses were used to analyze the prognostic value of C2. CIBERSORT was used to calculate the proportion of 22 kinds of tumor-infiltrating immune cells. Results. Significantly lower C2 expression was found at HCC compared to healthy controls, and C2 was associated with TNM stages. Higher C2 expression was significantly associated with better prognosis, and multivariate analysis showed that C2 was also an independent factor for the prognosis of HCC. Moreover, elevated CD4 T cells were found at HCC patients with higher $\mathrm{C} 2$ expression while the higher proportion of macrophage M0 cells was found in HCC patients with lower C2 expression. KEGG analysis showed that "cell cycle," "AMPK signaling pathway," and "PPAR signaling pathway" were enriched in HCC patients with higher $\mathrm{C} 2$ expression. Conclusion. C2 is a prognostic factor for HCC and may be used as a therapeutic target for future treatment of HCC.
\end{abstract}

\section{Introduction}

As one of the most common cancers and a leading cause of cancer-related death worldwide, hepatocellular carcinoma (HCC) continues to be a tremendous public burden on society [1]. Up to $50 \%$ of HCC patients are estimated to develop recurrence after resection partly due to limited amenable curative treatment options and rapid development of resistance [2]. A growing number of studies have indicated that tumor microenvironment (TME) plays important roles in almost every key aspect of HCC tumorigenesis, such as tumor initiation, progression, immune invasion, metastasis, recurrence, and resistance to therapy [3-5]. Therefore, understanding the interactions of stromal cells with cancer cells will help to develop effective strategies to conquer resistance and improve the therapeutic effect for HCC [6].
The complement system is a fundamental branch of innate immunity and could rapidly respond to invading pathogens by promoting cell lysis [7]. Remarkably, studies carried out over the last decade have shed new light on complement activation in the TME, which contributes to tumorpromoting and tumor-suppressing immune responses [8]. For example, complement component 7 (C7) and complement factor $\mathrm{H}(\mathrm{CFH})$ are found to be necessary for maintaining stemness of HCC cells as silence of C7 and CFH inhibits tumor-sphere formation and promotes cell differentiation while overexpression of them elevates stemness factor expression and cell growth in vivo [9]. C3 is required for the local and systemic immune responses against tumor in mice with G422 gliomas generated by photodynamic therapy (PDT), because knockout of $\mathrm{C} 3$ reduces the infiltration of immune cells and release of TNF- $\alpha$ and IFN- $\gamma$, which 
indicates a crucial role played by C3 in mediating antitumor immunity [10]. Interestingly, bilateral effect of complement component $5 \mathrm{a}(\mathrm{C} 5 \mathrm{a})$ on tumor progression has also been observed. High levels of C5a are related to tumorigenesis accompanied by reduced IFN- $\gamma$-producing CD 8 and CD 4 cells, while a low level of $\mathrm{C} 5 \mathrm{a}$ is associated with decreased tumor burden with increased IFN- $\gamma$-producing CD4 and CD8 $\mathrm{T}$ cells in mice with lymphoma [11]. These studies indicate entirely different roles played by each complement component on cancer development.

Complement component 2 (C2) is an important part of the complement system, and single nucleotide polymorphism (SNP) of C2 has been found to be significantly associated with HCC $[12,13]$. For example, C2 SNP rs9267665 is associated with the risk of developing HCC while rs 10947223 shows protective effects against HCC, indicating an important role played by $\mathrm{C} 2$ in HCC. However, up to now, little is known about the role and mechanism of $\mathrm{C} 2$ in $\mathrm{HCC}$, so in the present study, we aimed to explore the prognostic value of $\mathrm{C} 2$ and its correlation with tumor-infiltrating immune cells of TME in HCC patients.

\section{Materials and Methods}

2.1. Ethics Statement. All the data analyzed in the present study were attained from The Cancer Genome Atlas (TCGA) dataset, Gene Expression Omnibus (GEO) dataset, and the International Cancer Genome Consortium (ICGC) dataset, and informed consents had been gained from each patient before our study.

2.2. Acquisition of $m R N A$ Expression and Corresponding Clinical-Pathological Parameters from TCGA, GEO, and ICGC. In the present study, a total of three cohorts, including the TCGA HCC cohort, GSE14520 HCC cohort, and ICGC HCC cohort, were employed. mRNA expression and corresponding clinical-pathological parameters of the TCGA HCC cohort were downloaded from TCGA (https:// cancergenome.nih.gov/). In the TCGA HCC cohort, clinicalpathological parameters of 377 HCC patients, including gender, age, histologic grades, cirrhosis, TNM stage, status, and time of overall survival (OS), were attained. Meanwhile, mRNA expression of 374 HCC patients and 50 healthy controls was also downloaded. The GSE14520 HCC cohort was downloaded from GEO (https://www.ncbi.nlm.nih.gov/ geo/). In the GSE14520 HCC cohort, clinical-pathological parameters of 220 HCC patients, including gender, age, tumor size, cirrhosis, TNM staging, status, and time of OS, and mRNA expression of $220 \mathrm{HCC}$ patients and their corresponding adjacent normal tissues were available. mRNA expression and corresponding clinical-pathological parameters of the ICGC HCC cohort were got from the ICGC portal (https:// dcc.icgc.org/projects/LIRI-JP). In the ICGC HCC cohort, mRNA expression and clinical-pathological parameters of 232 HCC patients, including gender, age, TNM staging, status, and time of OS, were available. Basic demographic characteristics of TCGA, GSE14520, and ICGC HCC cohorts are summarized in Table 1.
2.3. CIBORSORT. An online tool, CIBERSORT, was used to calculate the proportion of 22 kinds of tumor-infiltrating immune cells with transcriptomic data (https://cibersort .stanford.edu). In CIBERSORT, relative fractions of 22 kinds of immune cells were deconvolved from the transcriptional expression of tumor samples basing on a referenced signature matrix by linear support vector regression.

2.4. Kyoto Encyclopedia of Genes and Genomes (KEGG) Analysis. The underlying mechanism of C2 in hepatocarcinogenesis was analyzed by KEGG analysis in the Database for Annotation, Visualization, and Integrated Discovery (DAVID) (https://david.ncifcrf.gov/summary.jsp). First, HCC patients of TCGA or GSE14520 or ICGC HCC cohort were divided into high $\mathrm{C} 2$ and low $\mathrm{C} 2$ groups according to the median $\mathrm{C} 2$ expression. Then, differentially expressed genes (DEGs) between the two groups were found with a cut-off value of $p<0.05$. Finally, KEGG pathways enriched by these DEGs were identified in DAVID, and a cut-off value of $p<0.05$ was considered as statistically significant.

2.5. Statistical Analysis. GraphPad Prism 6 (GraphPad Software, La Jolla, CA, USA) was used to carry out the statistical analyses. Data were presented as the median. Unpaired Student's $t$-tests or ANOVA tests were performed to compare the difference of $\mathrm{C} 2$ expression between HCC patients and healthy controls/adjacent normal tissues or among HCC patients with different histologic grades or TNM stages. Univariate and multivariate Cox regression analyses were carried out to analyze the prognostic value of $\mathrm{C} 2$ expression, and Kaplan-Meier analysis with a two-sided log-rank test was also performed to compare the OS of HCC patients with high or low C2 expression. Additional statistical analysis was performed with STAMP [14]. $p<0.05$ was considered as statistically significant.

\section{Results}

3.1. C2 Expression between HCC Patients and Healthy Controls/Adjacent Normal Tissues. We first analyzed the C2 expression between HCC patients and healthy controls/adjacent normal tissues of TCGA and GSE14520 HCC cohorts. As is shown in Figure 1, in the TCGA cohort, significantly lower C2 expression was found at HCC patients compared to healthy controls $(p<0.01$, Figure $1(\mathrm{a}))$. Similar results were also found in the GSE14520 cohort, in which significantly lower C2 expression was also found at HCC tissues compared to adjacent normal tissues $(p<0.001$, Figure $1(\mathrm{~b}))$. In short, the above results suggested that $\mathrm{C} 2$ expression of HCC was lower than that of healthy controls.

3.2. Association of C2 Expression with Clinical-Pathological Parameters of HCC Patients. After reduced C2 expression was found in HCC patients, we next analyzed the association of C2 expression with clinical-pathological parameters. As is shown in Figure 2, in the TCGA cohort, C2 expression was remarkably correlated with TNM stages. HCC patients who were in more advanced TNM stages expressed lower C2 expression $(p=0.02$, Figure 2(a)). Besides, HCC patients who were in more decreased differentiation tended to express 
TABLE 1: Basic characteristics of HCC patients from the TCGA, GSE14520, and ICGC HCC cohorts.

\begin{tabular}{lccc}
\hline Variables & TCGA cohort $(N=377)$ & GSE14520 cohort $(N=220)$ & ICGC cohort $(N=232)$ \\
\hline Gender (male/female) & $255 / 122$ & $190 / 30$ & $171 / 61$ \\
Age $($ years, median) & $60(16-90)$ & $50(21-77)$ & $69(31-89)$ \\
Cirrhosis $($ yes/no/NA) & $81 / 137 / 159$ & $202 / 18$ & $\mathrm{NA}$ \\
Histologic grade $(\mathrm{G} 1 / \mathrm{G} 2 / \mathrm{G} 3 / \mathrm{G} 4 / \mathrm{NA})$ & $\mathrm{NA}$ & $\mathrm{NA}$ \\
TNM stage (I/II/III/IV/NA) & $55 / 180 / 124 / 13 / 5$ & $93 / 77 / 48 /-/ 2$ & $36 / 106 / 71 / 76$ \\
Main tumor size $(>/ \leq 5 \mathrm{~cm})$ & $175 / 87 / 86 / 5 / 24$ & $80 / 140$ & $\mathrm{NA}$ \\
Overall survival status (alive/dead) & $\mathrm{NA}$ & $136 / 84$ & $189 / 43$ \\
Overall survival time (months, median) & $245 / 132$ & $51.65(1.8-67.4)$ & $26.0(0.3-72)$ \\
\hline
\end{tabular}

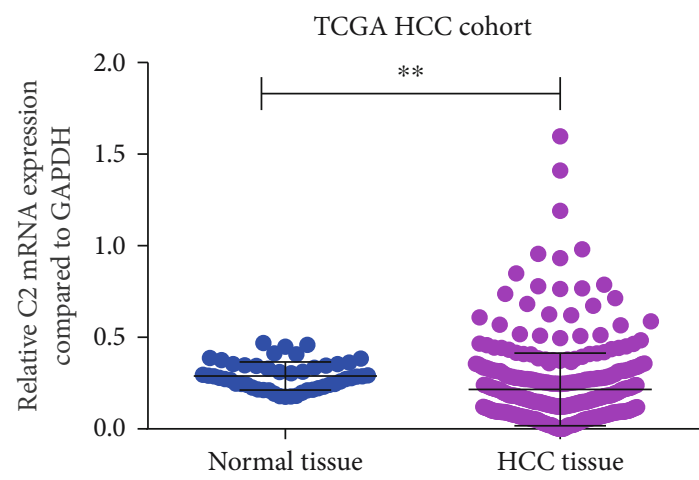

(a)

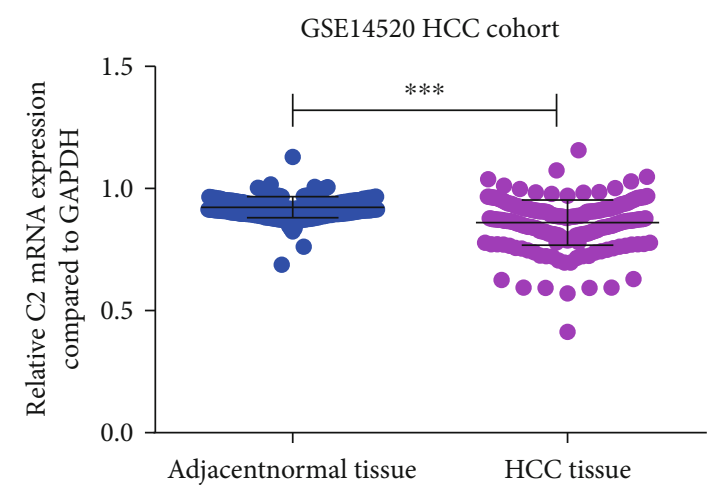

(b)

FIGURE 1: C2 expression between HCC patients and healthy controls/adjacent normal tissues. Expression of C2 in HCC patients and healthy controls of the TCGA cohort (a); expression of C2 in HCC patients and adjacent normal tissues of the GSE14520 cohort (b).

lower C2 expression too, but the difference was not significant $(p=0.18$, Figure 2(b)). Similar results were also found at ICGC and GSE14520 HCC cohorts. In the ICGC cohort, HCC patients who were in more advanced TNM stages expressed lower C2 expression $(p=0.004$, Figure 2(c)). In the GSE14520 cohort, HCC patients who were in more advanced TNM stages expressed lower C2 expression $(p<0.0001$, Figure 2(d)). Moreover, C2 expression of HCC patients with tumor size $\geq 5 \mathrm{~cm}$ was lower than that of HCC patients with tumor size $\leq 5 \mathrm{~cm} \quad(p=0.002$, Figure 2(e)). Taken together, C2 expression was significantly associated with TNM stages in HCC patients, and the higher the TNM stages, the lower the C2 expression.

3.3. Prognostic Value of C2 in HCC Patients. Having found that $\mathrm{C} 2$ expression was significantly associated with clinical-pathological parameters in HCC patients, we next analyzed the prognostic value of $\mathrm{C} 2$ in HCC patients. As is shown in Figure 3, C2 expression was significantly associated with the prognosis of HCC patients. In the TCGA cohort, univariate Cox analysis showed that higher C2 expression was significantly associated with better OS of HCC patients $(\mathrm{HR}=0.66,95 \% \mathrm{CI}: 0.47-0.93, p=0.02$, Figure 3(a)). Moreover, multivariate analysis also showed that $\mathrm{C} 2$ expression was independently associated with OS of HCC patients after adjusting for gender, age, cirrhosis, histologic grade, and TNM stage $(\mathrm{HR}=0.68,95 \% \mathrm{CI}: 0.47-0.99, p=0.04$, Table 2). In the GSE14520 cohort, univariate Cox indicated that higher $\mathrm{C} 2$ expression was also significantly associated with better OS of HCC patients ( $\mathrm{HR}=0.56,95 \% \mathrm{CI}$ : 0.36 $0.87, p=0.01$, Figure $3(\mathrm{~b}))$, but multivariate analysis showed that $\mathrm{C} 2$ expression was not independently associated with OS of HCC patients after adjusting for gender, age, cirrhosis, main tumor size, and TNM stage ( $\mathrm{HR}=0.73$, 95\% CI: 0.47 $1.16, p=0.18$, Table 3 ). In the ICGC cohort, univariate Cox suggested that higher $\mathrm{C} 2$ expression was significantly related with better OS ( $\mathrm{HR}=0.46,95 \% \mathrm{CI}$ : $0.25-0.86, p=0.02$, Figure 3(c)), and multivariate analysis also showed that $\mathrm{C} 2$ expression was independently associated with OS of HCC patients after adjusting for gender, age, and TNM stage $(\mathrm{HR}=0.73,95 \% \mathrm{CI}: 0.27-0.96, p=0.04$, Table 4$)$. In a word, $\mathrm{C} 2$ may play an important role in HCC suppression.

3.4. Correlation of C2 Expression with Tumor-Infiltrating Immune Cells in HCC Patients. Increasing studies had proved an immunoregulatory effect of complement on TME, and interactions between complement and tumor-infiltrating immune cells contribute to the development and progression of many kinds of cancers [8]. So, we further analyzed the correlation of $\mathrm{C} 2$ expression with tumor-infiltrating immune cells in HCC patients. As is shown in Figure 4, C2 expression was significantly associated with tumor-infiltrating immune cells in HCC patients. In the TCGA cohort, significantly higher proportions of resting CD4 memory $\mathrm{T}$ cells and macrophage M1 cells were found in HCC patients with higher C2 expression, while significantly higher proportions 


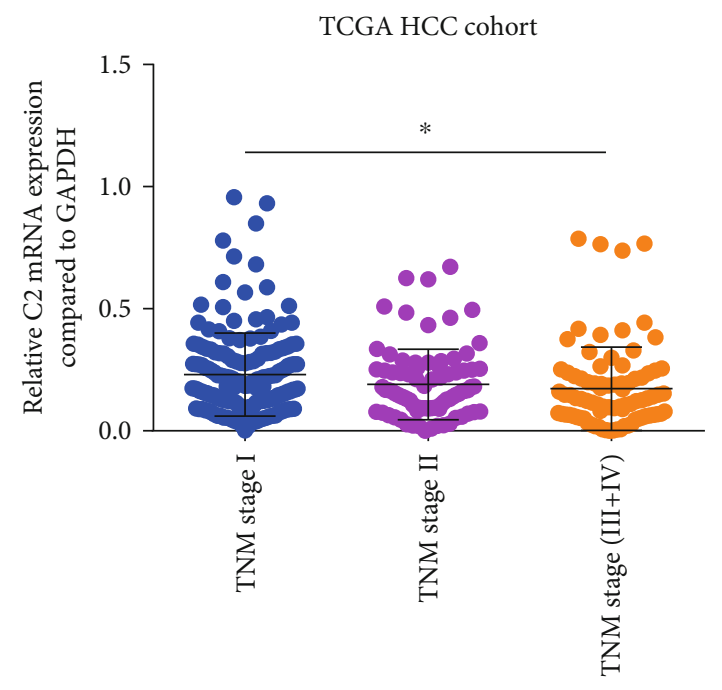

(a)

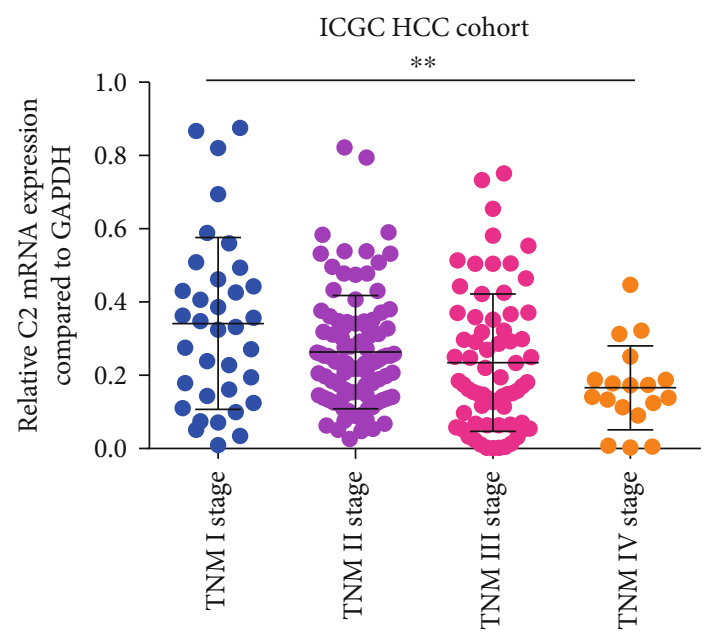

(c)
TCGA HCC cohort

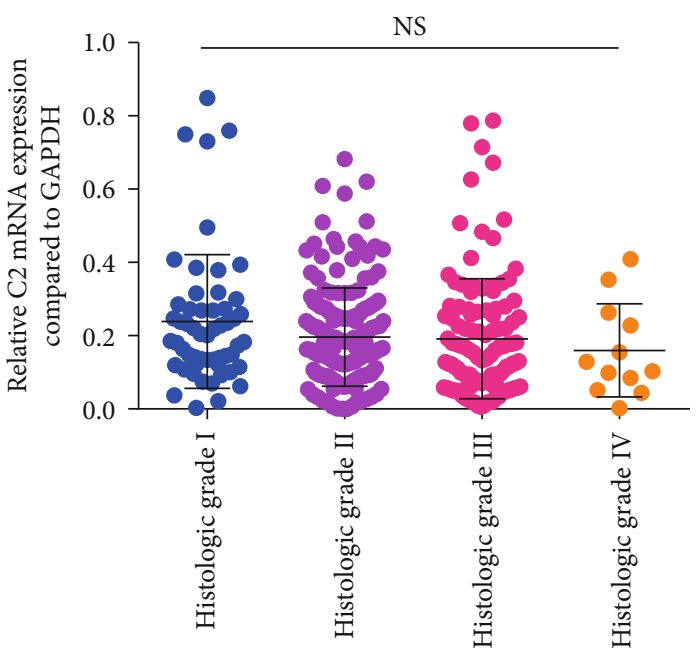

(b)

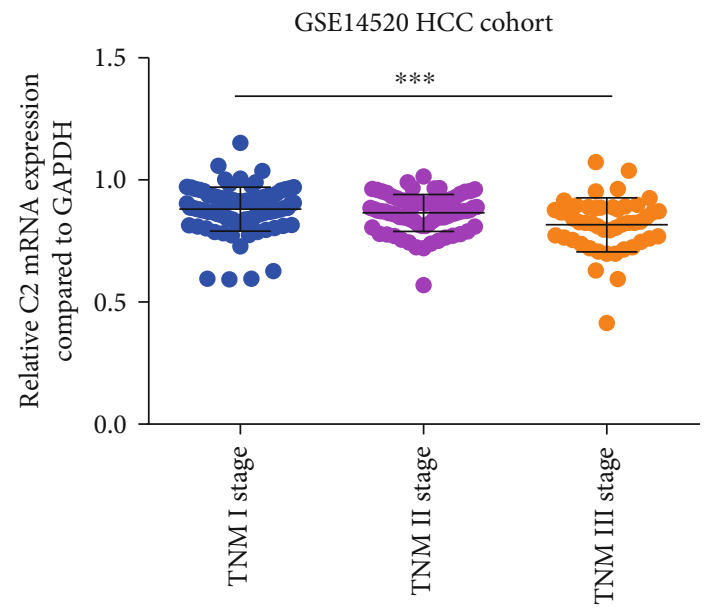

(d)

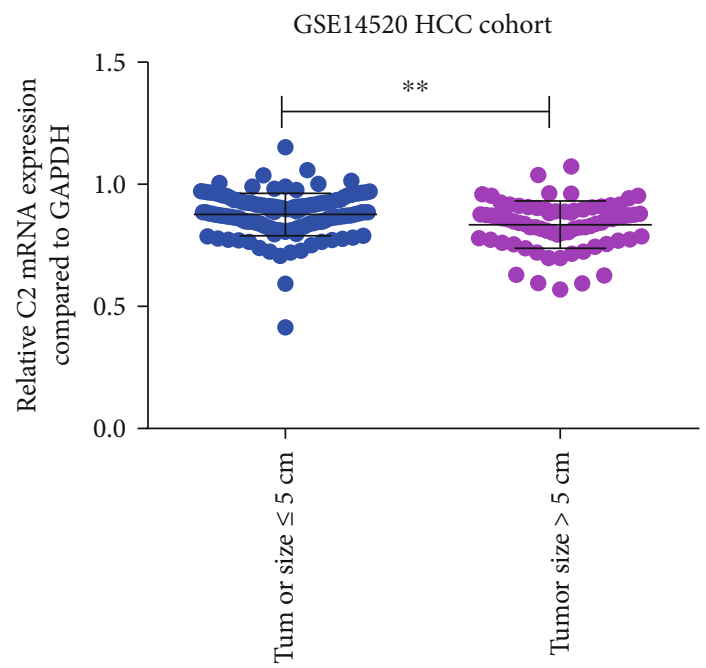

(e)

FIGURE 2: Association of C2 expression of HCC patients with clinical-pathological parameters. Association of C2 expression with TNM stages and histologic grades of TCGA cohort $(\mathrm{a}, \mathrm{b})$; association of C2 expression with TNM stages of ICGC cohort (c); association of C2 expression with TNM stages and main tumor size of cohort $(\mathrm{d}, \mathrm{e})$. 
TCGA-HCC cohort

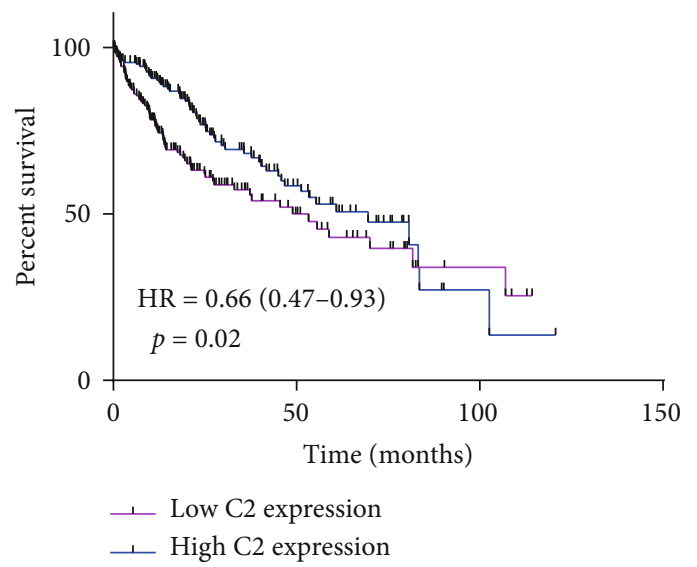

(a)
GSE14520-HCC cohort

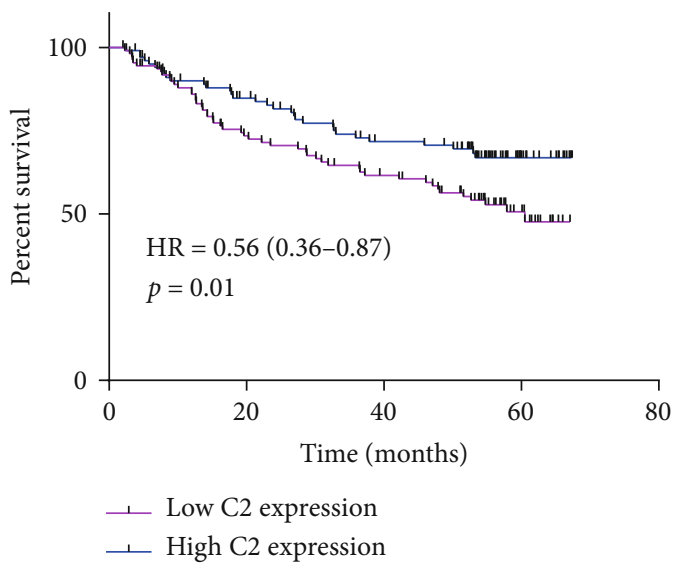

(b)

ICGC-HCC cohort

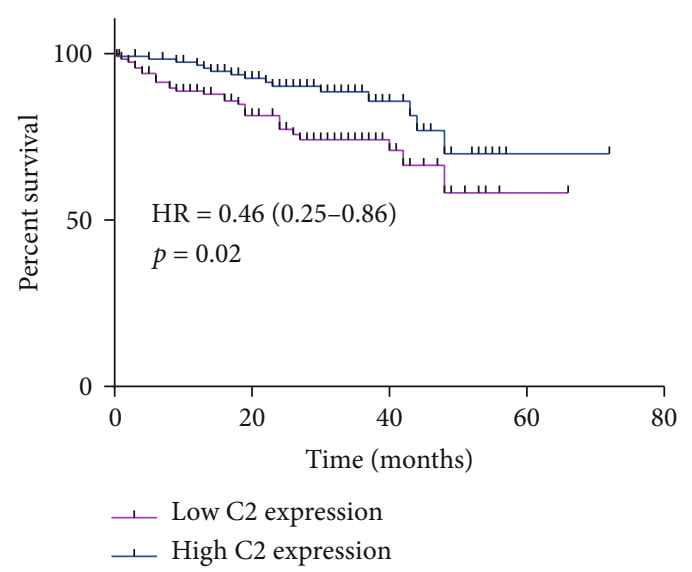

(c)

Figure 3: Prognostic value of C2 in HCC patients. Kaplan-Meier analysis of overall survival time of HCC patients with high C2 and low C2 expression in the TCGA cohort (a); Kaplan-Meier analysis of overall survival time of HCC patients with high C2 and low C2 expression in the GSE14520 cohort (b); Kaplan-Meier analysis of overall survival time of HCC patients with high C2 and low C2 expression in the ICGC cohort (c).

TABLE 2: Univariate and multivariate analyses of C2 expression for overall survival in HCC patients of the TCGA cohort.

\begin{tabular}{|c|c|c|c|c|c|c|}
\hline \multirow{2}{*}{ Variables } & \multicolumn{3}{|c|}{ Univariate analysis } & \multicolumn{3}{|c|}{ Multivariate analysis } \\
\hline & Hazard ratio & $95 \% \mathrm{CI}$ & $p$ value & Hazard ratio & $95 \% \mathrm{CI}$ & $p$ value \\
\hline Gender (male vs. female) & 0.82 & $0.57-1.16$ & 0.26 & & & \\
\hline Age (>60 vs. $\leq 60)$ & 1.25 & $0.88-1.77$ & 0.21 & & & \\
\hline Cirrhosis (yes vs. no) & 0.83 & $0.48-1.42$ & 0.49 & & & \\
\hline Histologic grade $(\mathrm{G} 3+\mathrm{G} 4$ vs. $\mathrm{G} 1+\mathrm{G} 2)$ & 1.12 & $0.78-1.61$ & 0.54 & & & \\
\hline TNM stage (III+IV vs. I+II) & 2.43 & $1.69-3.55$ & $0.000^{*}$ & 2.38 & $1.64-3.46$ & $0.000^{*}$ \\
\hline C2 (high vs. low) & 0.66 & $0.47-0.93$ & $0.02^{*}$ & 0.68 & $0.47-0.99$ & $0.04^{*}$ \\
\hline
\end{tabular}

of macrophage M0 cells, activated CD4 memory cells, and plasma cells were found at HCC patients with lower C2 expression (all $p<0.05$, Figure 4(a)). In the GSE14520 cohort, significantly higher proportions of resting mast cells, follicular helper T cells, and resting CD4 memory T cells were found in HCC patients with higher C2 expression, while significantly higher proportions of macrophage M0 cells, acti- vated mast cells, and plasma cells were found at HCC patients with lower C2 expression (all $p<0.05$, Figure $4(\mathrm{~b})$ ). In the ICGC cohort, significantly higher proportions of resting CD4 memory T cells were found in HCC patients with higher C2 expression, while significantly higher proportions of macrophage M0 cells, activated CD4 memory T cells, and Treg cells were found at HCC patients with lower C2 
TABLE 3: Univariate and multivariate analysis of C2 expression for overall survival in HCC patients of the GSE14520 cohort.

\begin{tabular}{|c|c|c|c|c|c|c|}
\hline \multirow{2}{*}{ Variables } & \multicolumn{3}{|c|}{ Univariate analysis } & \multicolumn{3}{|c|}{ Multivariate analysis } \\
\hline & Hazard ratio & $95 \% \mathrm{CI}$ & $p$ value & Hazard ratio & $95 \% \mathrm{CI}$ & $p$ value \\
\hline Gender (male vs. female) & 1.68 & $0.81-3.49$ & 0.16 & & & \\
\hline Age (>50 vs. $\leq 50)$ & 1.0 & $0.65-1.53$ & 0.99 & & & \\
\hline Cirrhosis (yes vs. no) & 4.57 & $1.13-18.62$ & $0.03^{*}$ & 3.66 & $0.89-14.98$ & 0.07 \\
\hline Main tumor size ( $>5 \mathrm{~cm}$ vs. $\leq 5 \mathrm{~cm}$ ) & 1.97 & $1.28-3.30$ & $0.002^{*}$ & 1.18 & $0.69-2.01$ & 0.56 \\
\hline TNM stage (III+IV vs. I+II) & 3.43 & $2.20-6.11$ & $0.000^{*}$ & 2.38 & $1.64-3.46$ & $0.000^{*}$ \\
\hline C2 (high vs. low) & 0.56 & $0.36-0.87$ & $0.01^{*}$ & 0.73 & $0.47-1.16$ & 0.18 \\
\hline
\end{tabular}

TABLE 4: Univariate and multivariate analyses of C2 expression for overall survival in HCC patients of the ICGC cohort.

\begin{tabular}{|c|c|c|c|c|c|c|}
\hline \multirow{2}{*}{ Variables } & \multicolumn{3}{|c|}{ Univariate analysis } & \multicolumn{3}{|c|}{ Multivariate analysis } \\
\hline & Hazard ratio & $95 \% \mathrm{CI}$ & $p$ value & Hazard ratio & $95 \% \mathrm{CI}$ & $p$ value \\
\hline Gender (male vs. female) & 0.51 & $0.28-0.97$ & $0.04^{*}$ & 0.43 & $0.23-0.81$ & $0.01^{*}$ \\
\hline Age (>70 vs. $\leq 70$ ) & 1.06 & $0.58-1.94$ & 0.84 & & & \\
\hline TNM stage (III+IV vs. I+II) & 2.38 & $1.30-4.36$ & $0.004^{*}$ & 2.59 & $1.39-4.80$ & $0.003^{*}$ \\
\hline C2 (high vs. low) & 0.46 & $0.25-0.86$ & $0.02^{*}$ & 0.73 & $0.27-0.96$ & $0.04^{*}$ \\
\hline
\end{tabular}

expression (all $p<0.05$, Figure $4(\mathrm{c})$ ). Taken together, C2 expression was significantly associated with CD4 memory $\mathrm{T}$ cells and macrophage M0 cells in HCC patients from all these three HCC cohorts.

3.5. Molecular Mechanisms of C2 in HCC. KEGG analysis was performed to explore the underlying biological mechanism by which $\mathrm{C} 2$ influenced the prognosis of HCC. As is shown in Figure 5, in the TCGA cohort, KEGG pathways, such as "cell cycle," "ubiquitin-mediated proteolysis," "complement and coagulation cascades," "spliceosome," and "RNA transport," were most significantly enriched in HCC patients with higher C2 expression compared to HCC patients with lower C2 expression (all $p<0.001$, Figure 5(a)). In the GSE14520 cohort, KEGG pathways, such as "complement and coagulation cascades," "carbon metabolism," "biosynthesis of amino acids," "peroxisome," and "ribosome," were most significantly enriched in HCC patients with higher C2 expression (all $p<0.001$, Figure 5(b)). In the ICGC cohort, KEGG pathways, such as "ribosome," "cell cycle," "complement and coagulation cascades," "spliceosome," and "RNA transport," were most significantly enriched in HCC patients with higher C2 expression (all $p<0.001$, Figure 5(c)). In total, as is shown in Table 5, 17 significant KEGG pathways, such as "cell cycle," "complement and coagulation cascades," "AMPK signaling pathway," and "PPAR signaling pathway," overlapped in the three HCC cohorts, indicating that C2 may influence the prognosis of HCC by regulation of these 17 overlapped KEGG pathways.

\section{Discussion}

HCC is one of the most malignant kinds of cancer worldwide, and TME has been found to play important roles of recurrence and resistance to therapy of $\operatorname{HCC}[8,15]$. Increasing studies have proved an immunoregulatory effect of complement on TME, and interactions between complement and TME contribute to the development and progression of many kinds of cancers [8]. C2 is an important part of the complement system, and SNP of C2 has been found to be significantly associated with HCC $[12,13]$. In the present study, we found that $\mathrm{C} 2$ was associated with the prognosis of HCC and explored the relevant underlying mechanism.

Previously, Imamura et al. have found that higher expression of the C5a receptor from breast cancer tissues is significantly associated with clinical parameters, such as larger tumor size, advanced histologic grade, lymph node metastasis, higher TNM stages, and poorer prognosis [16]. Lin et al. have also observed that higher tissue C3 expression is significantly associated with better prognosis of non-smallcell lung cancer (NSCLC) patients, indicating an important role played by C3 in NSCLC suppression [17]. Similarly, in our study, lower expression of $\mathrm{C} 2$ was found at HCC patients, and C2 expression was significantly associated with TNM stages and better OS. Besides, C2 expression was independently associated with OS of HCC patients in TCGA and ICGC HCC cohorts, but it was not in the GSE14520 cohort. The reason for the difference in the predictive value of $\mathrm{C} 2$ expression in different HCC cohorts may be that some pathological parameters among the three HCC cohorts were different. For example, in the TCGA HCC cohort, cirrhosis was not associated with OS, but in the GSE14520 HCC cohort, cirrhosis and main tumor size were significantly associated with OS. Moreover, in the TCGA and GSE1420 HCC cohorts, gender was not associated with OS, but in the ICGC HCC cohort, gender was associated with OS. The difference of cirrhosis, main tumor size, and gender among the three HCC cohorts may result in the difference of factors used 
TCGA HCC cohort

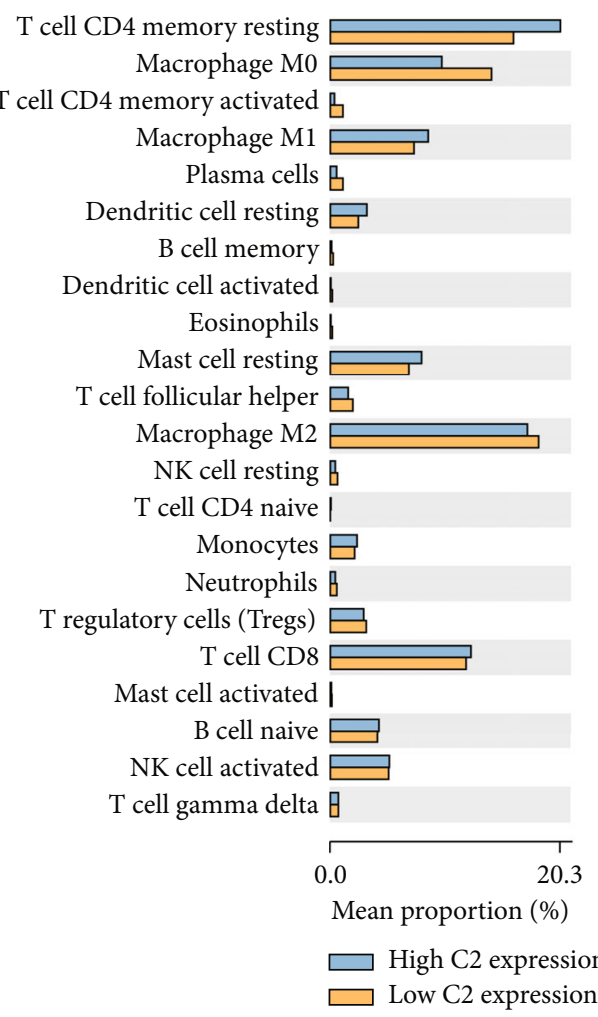

\section{ICGC HCC cohort}

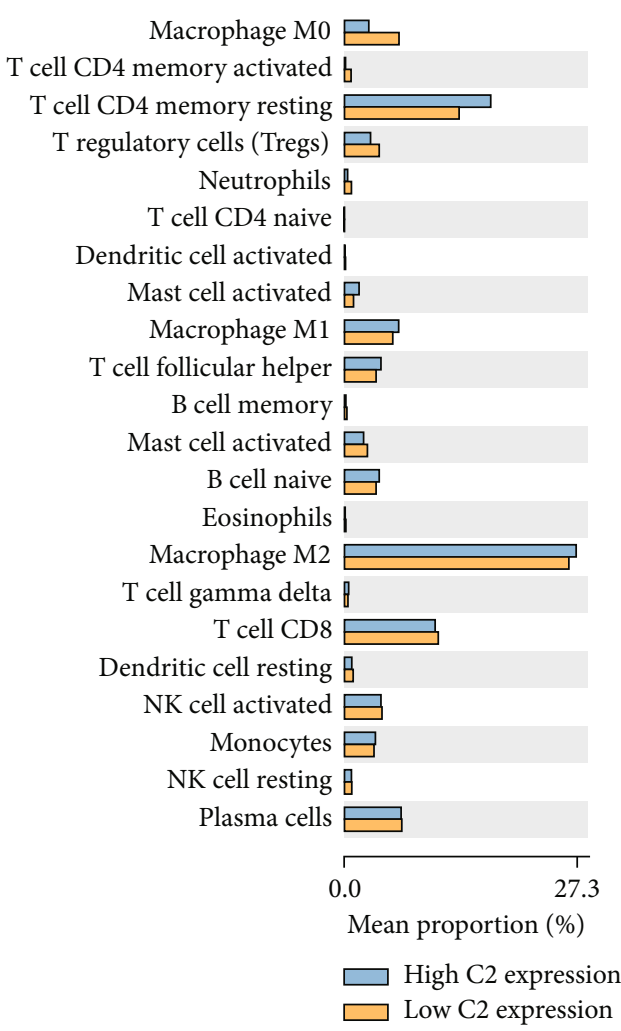

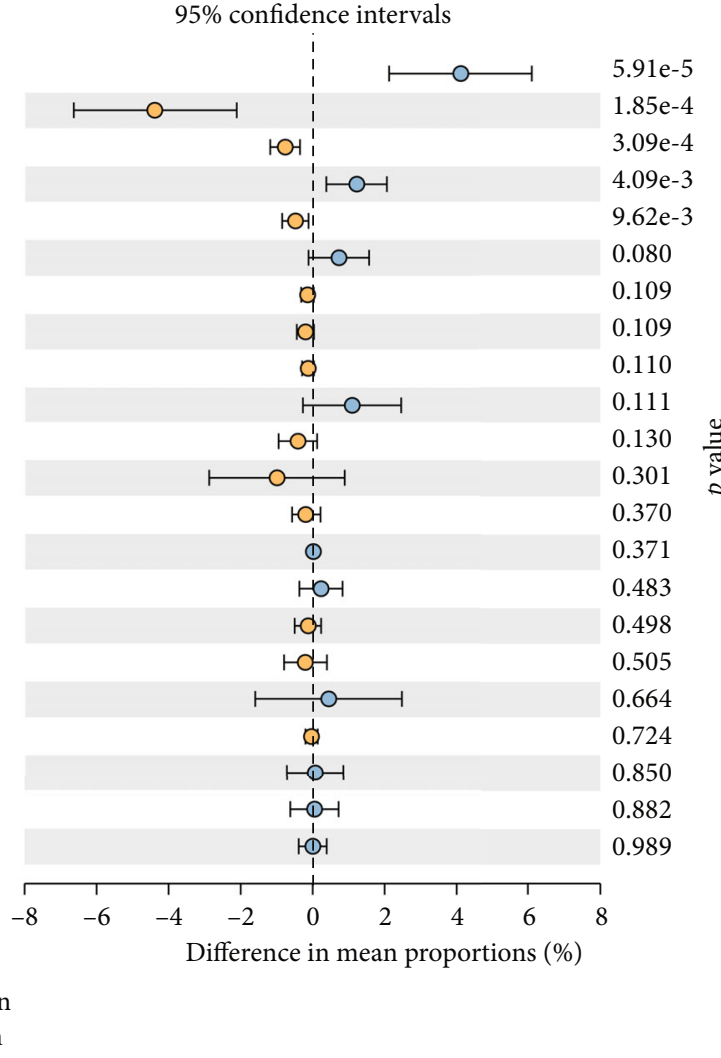

(a)

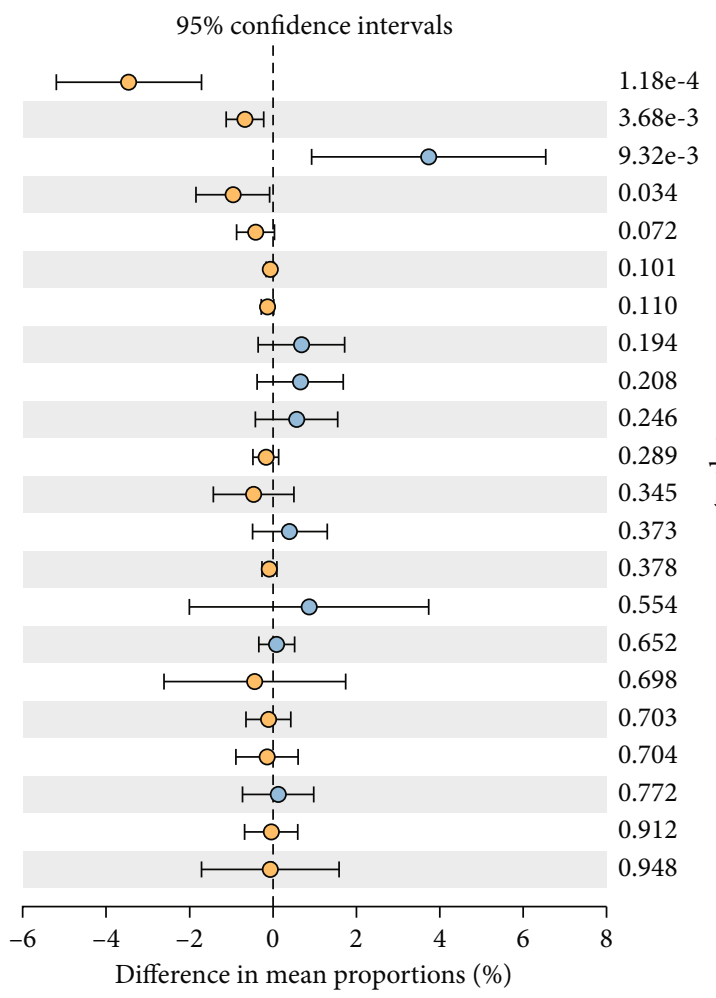

(b)

FIGURE 4: Continued. 


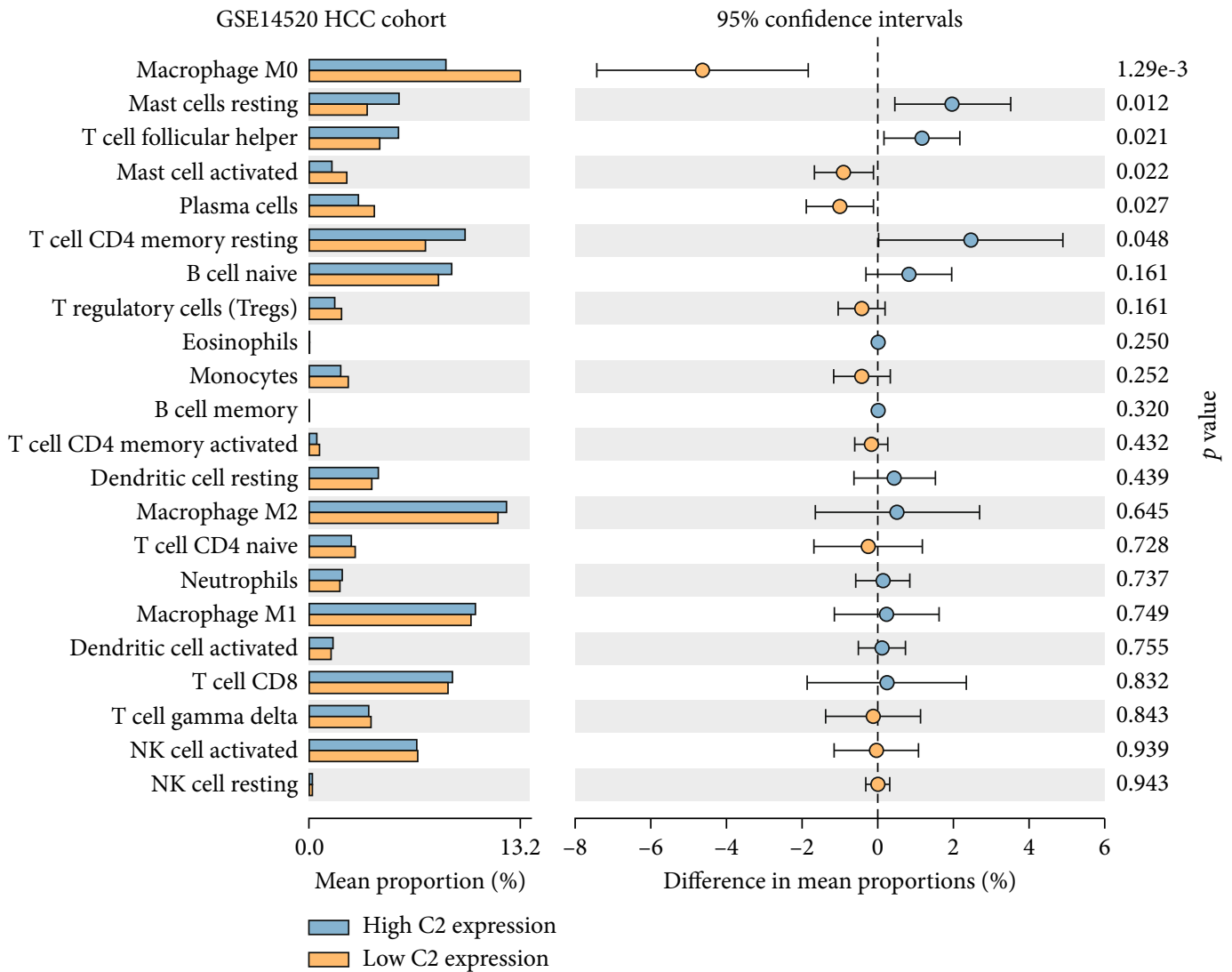

(c)

FIGURE 4: Correlation of C2 expression with tumor-infiltrating immune cells in HCC patients of the TCGA cohort (a), GSE14520 cohort (b), and ICGC cohort (c).

for multivariate analysis, thus leading to the difference of the predictive value of $\mathrm{C} 2$ expression for OS. In a word, C2 expression was associated with OS of HCC patients, but it still needed external and multicenter prospective cohorts with large sample sizes to validate whether $\mathrm{C} 2$ expression could be an independent prognostic factor for OS.

A series of studies have proved that the complement system takes part in the regulation of TME, and interaction of complement with tumor-infiltrating immune cells plays an important role in the development and progression of many kinds of cancers [8]. Tumor cell-derived C5a can recruit and differentiate myeloid-derived suppressor cells (MDSCs) in TME, which could protect tumor cells against the immune system and immunotherapy and promote tumor progression by inhibiting $\mathrm{T}$ cell responses and promoting the generation of Tregs [18]. Moreover, blockade of C5aR can reduce the number of MDSCs [19]. Lin et al. have also showed that higher tissue C3 expression was positively correlated with higher numbers of tumor-infiltrating CD4 T cells and CD8 $\mathrm{T}$ cells, which may also contribute to tumor suppression by $\mathrm{C} 3$, as higher $\mathrm{C} 3$ expression predicts better prognosis in NSCLC patients [17]. In the present study, elevated CD4 T cells were found at HCC patients with higher $\mathrm{C} 2$ expression while a higher proportion of macrophage $\mathrm{M} 0$ cells was found in HCC patients with lower C2 expression in all three HCC cohorts. Garnelo et al. have showed that the degree of infil- trated T cells and B cells of tumor tissues significantly relates to the improved prognosis of HCC patients [20]. CD4+ T cells can improve antitumor immune responses by producing cytokines which are important for the activation of CD8+ T cells and B cells. Imai et al. have found that CD4+ $\mathrm{T}$ cells are important for the formation and maintenance of polyfunctionality of cytotoxic CD8+ T cells, which is a key determinant of the success of immunological control of tumor growth [21]. Moreover, the important role of virus control and antitumor immunity played by $\mathrm{CD} 4+\mathrm{T}$ cellmediated cytotoxicity is being increasingly recognized. Fu et al. have found that circulating and tumor-infiltrating CD4+ cytotoxic T cells decrease in HCC patients with advanced stages, and loss of CD4+ cytotoxic T cells is associated with a high mortality rate and reduced survival time [22]. Hsiao et al. have observed that the higher number of macrophage M0 cells is significantly associated with poorer prognosis of HCC patients [23]. Macrophages can be recruited into HCC tissue to become tumor-associated macrophages (TAMs) by upregulation of HMGB1 and then take part in the cancer progression and metastasis [24]. TAMs locate in the stroma of HCC tissue and are polarized toward the M2 phenotype. A lot of studies have showed that TAMs can promote tumor proliferation, angiogenesis, invasion, and metastasis [25]. For example, Yeung et al. have showed that M2 macrophages are associated with a poor prognosis 
Cell cycle

Ubiquitin mediated proteolysis Complement and coagulation cascades

Spliceosome

RNA transport

Carbon metabolism

Salmonella infection

Cholesterol metabolism

Insulin signaling pathway

Huntington disease

Human T-cell leukemia virus 1 infection

Pyrimidine metabolism

Endocytosis

Amino sugar and nucleotide sugar metabolism

Insulin resistance

Small cell lung cancer mTOR signaling pathway

Colorectal cancer

Glycolysis / gluconeogenesis

Oxidative phosphorylation

Hepatocellular carcinoma

Pyruvate metabolism

RNA degradation

Cellular senescene Lysosome mRNA surveillance pathway Oocyte meiosis

Shigellosis

HIF-1 signaling pathway Thermogenesis

$$
0
$$

Complement and coagulation cascades Carbon metabolism Biosynthesis of amino acids Peroxisome Ribosome

Proteoglycans in cancer Alanine, aspartate and glutamate metabolism

PPAR signaling pathway

Valine, leucine and isoleucine degradation Pentose phosphate pathway

Cholesterol metabolism

HIF-1 signaling pathway Bile secretion

AMPK signaling pathway

Fatty acid degradation

Glycine, serine and threonine metabolism

Glycolysis / gluconeogenesis

Glyoxylate and dicarboxylate metabolism Cell cycle

Longevity regulating pathway - multiple species

Chemical carcinogenesis

Fructose and mannose metabolism

Vibrio cholerae infection

Central carbon metabolism in cancer

Propanoate metabolism

Fatty acid metabolism

Cysteine and methionine metabolism Insulin resistance

One carbon pool by folate Insulin signaling pathway
TCGA HCC cohort

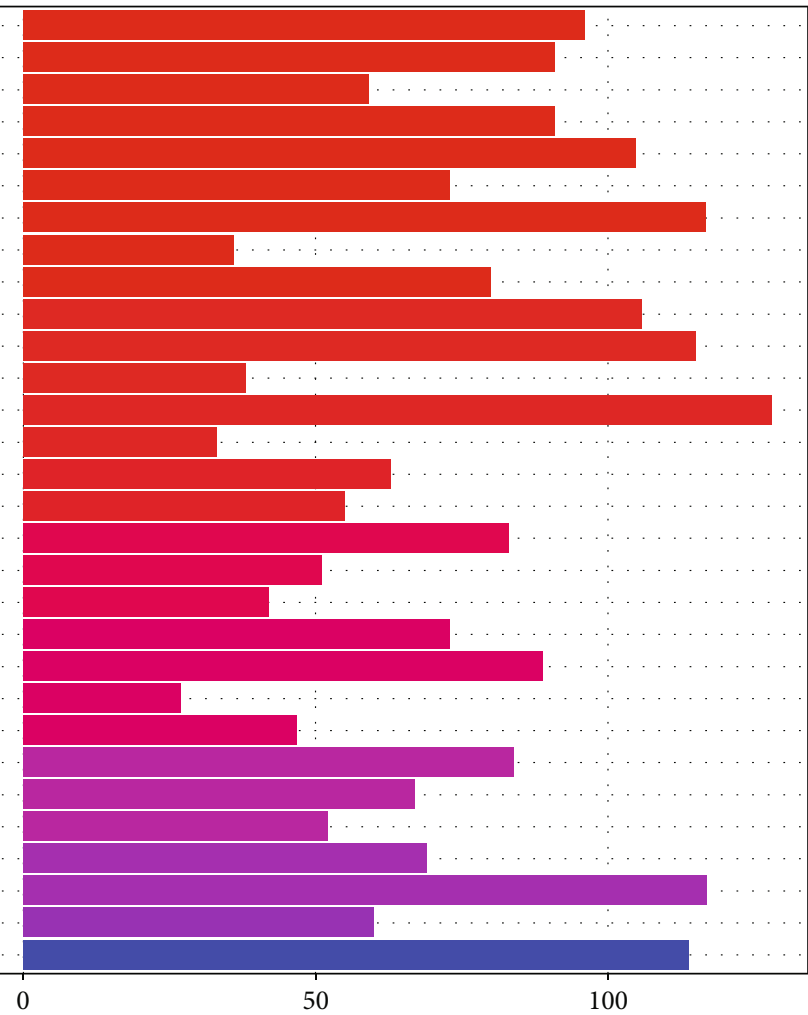

(a)

GSE14520 HCC cohort

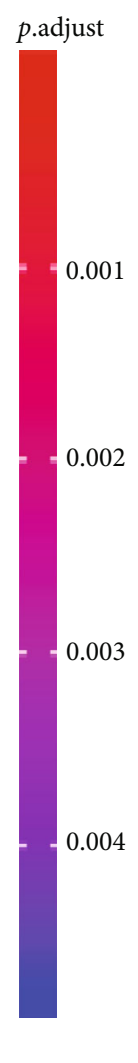

p.adjust

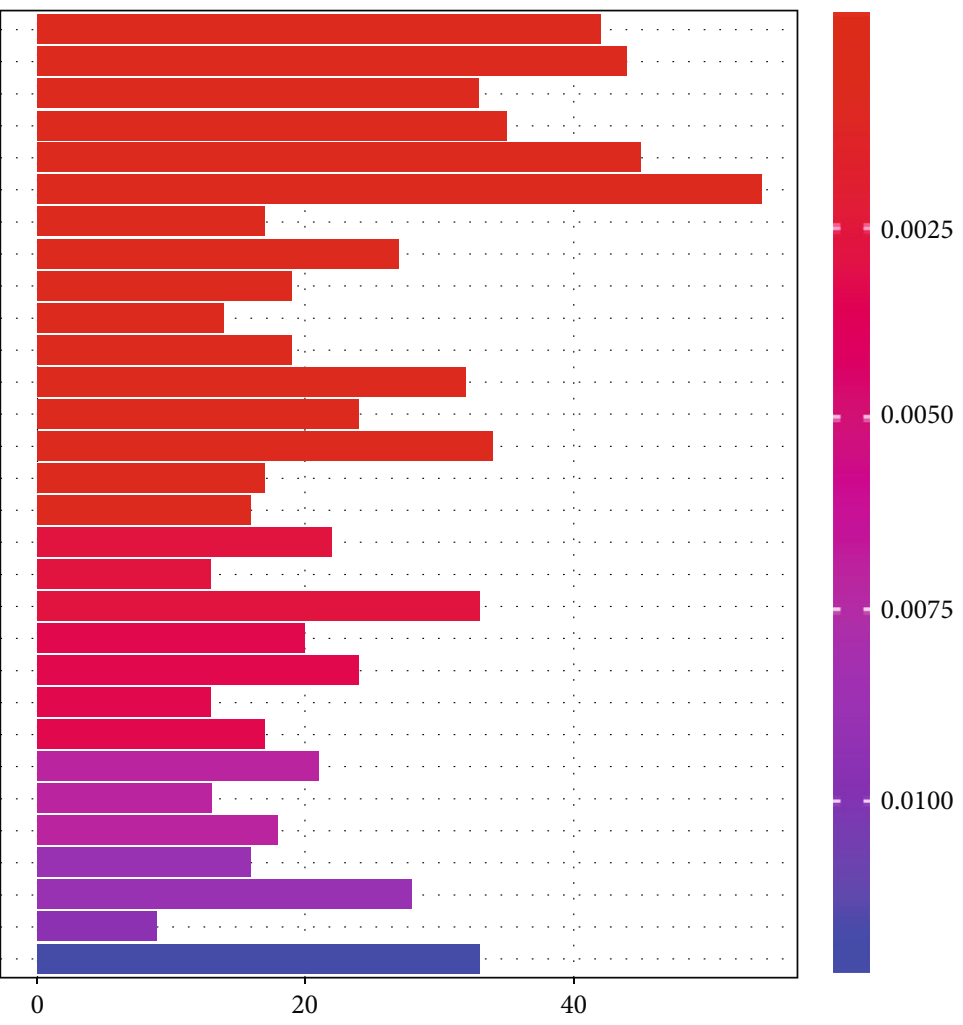

(b)

Figure 5: Continued. 


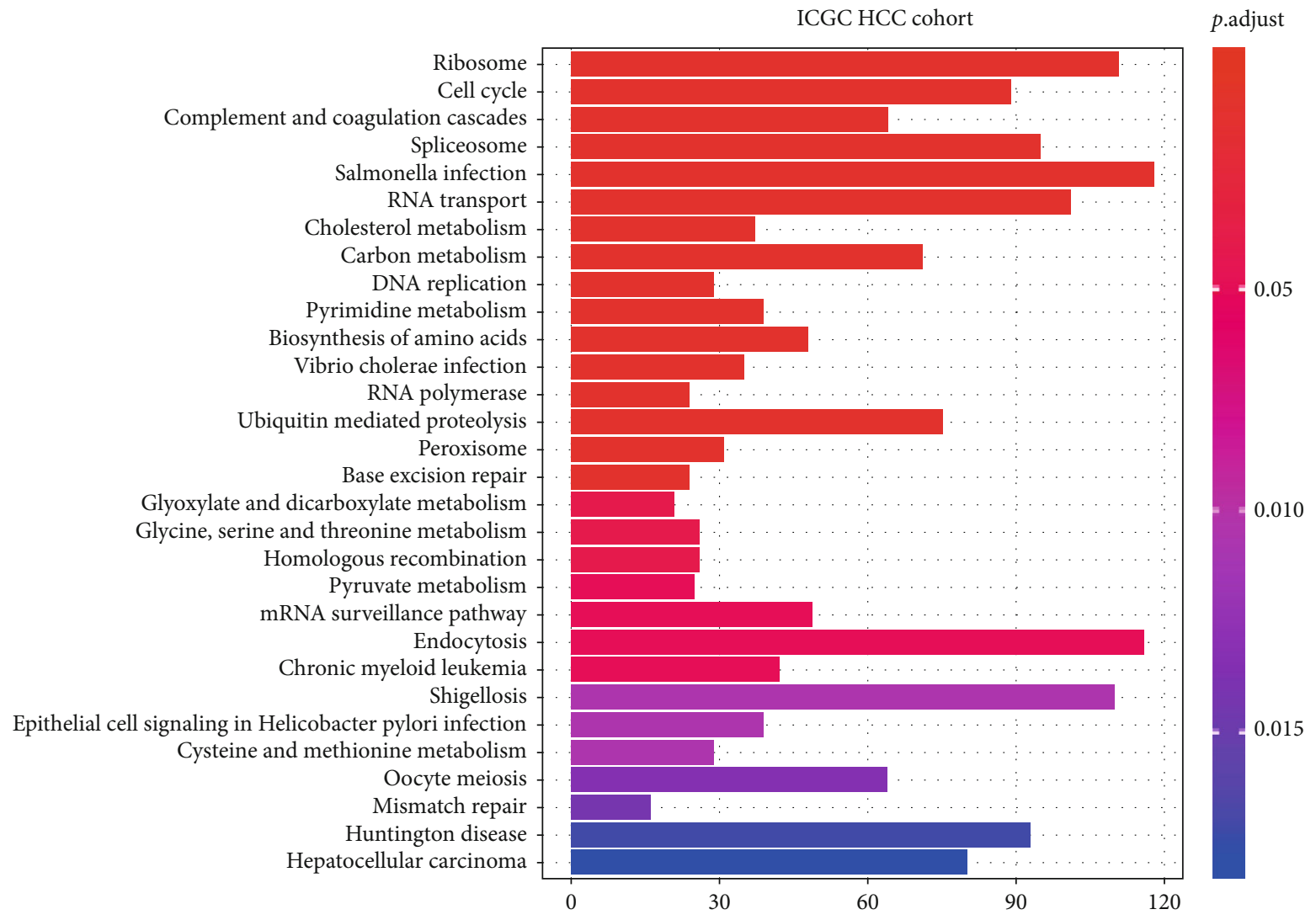

(c)

FIGURE 5: The most significant 30 KEGG pathways enriched in HCC patients with higher C2 expression compared to HCC patients with lower C2 expression of the TCGA cohort (a), GSE14520 cohort (b), and ICGC cohort (c).

TABLE 5: Overlap of significant KEGG pathways enriched in HCC patients with higher C2 expression from the TCGA, GSE14520, and ICGC HCC cohorts.

\begin{tabular}{|c|c|c|c|c|c|c|c|}
\hline \multirow{2}{*}{ ID } & \multirow{2}{*}{ Description } & \multicolumn{2}{|c|}{ TCGA cohort } & \multicolumn{2}{|c|}{ GSE14520 cohort } & \multicolumn{2}{|c|}{ ICGC cohort } \\
\hline & & Count & p.adjust & Count & p.adjust & Count & p.adjust \\
\hline hsa04110 & Cell cycle & 96 & 0.000 & 33 & 0.003 & 89 & 0.000 \\
\hline hsa04610 & Complement and coagulation cascades & 59 & 0.000 & 42 & 0.000 & 64 & 0.000 \\
\hline hsa04152 & AMPK signaling pathway & 61 & 0.023 & 34 & 0.001 & 58 & 0.031 \\
\hline hsa03320 & PPAR signaling pathway & 42 & 0.014 & 27 & 0.000 & 40 & 0.025 \\
\hline hsa04910 & Insulin signaling pathway & 80 & 0.000 & 28 & 0.008 & 66 & 0.026 \\
\hline hsa01200 & Carbon metabolism & 73 & 0.000 & 44 & 0.000 & 71 & 0.000 \\
\hline hsa00630 & Glyoxylate and dicarboxylate metabolism & 20 & 0.012 & 13 & 0.002 & 21 & 0.004 \\
\hline hsa00620 & Pyruvate metabolism & 27 & 0.001 & 13 & 0.017 & 25 & 0.006 \\
\hline hsa01230 & Biosynthesis of amino acids & 42 & 0.011 & 33 & 0.000 & 48 & 0.000 \\
\hline hsa00410 & Beta-alanine metabolism & 19 & 0.039 & 11 & 0.021 & 19 & 0.030 \\
\hline hsa00270 & Cysteine and methionine metabolism & 29 & 0.017 & 16 & 0.008 & 29 & 0.012 \\
\hline hsa05110 & Vibrio cholerae infection & 31 & 0.006 & 17 & 0.004 & 35 & 0.000 \\
\hline hsa00010 & Glycolysis/gluconeogenesis & 42 & 0.001 & 22 & 0.002 & 36 & 0.029 \\
\hline hsa04979 & Cholesterol metabolism & 36 & 0.000 & 19 & 0.001 & 37 & 0.000 \\
\hline hsa00280 & Valine, leucine, and isoleucine degradation & 27 & 0.042 & 19 & 0.000 & 27 & 0.029 \\
\hline hsa00640 & Propanoate metabolism & 21 & 0.027 & 54 & 0.000 & 20 & 0.041 \\
\hline hsa00260 & Glycine, serine, and threonine metabolism & 23 & 0.048 & 16 & 0.001 & 26 & 0.004 \\
\hline
\end{tabular}


in HCC by promoting tumor growth and invasiveness through CCL22-induced epithelial-mesenchymal transition (EMT) [26]. Besides, tumor-suppressive M1 macrophages were found to be enriched in HCC patients with higher C2 expression in the TCGA cohort, which may contribute to better prognosis of HCC patients as studies have showed that M1 macrophages are involved in killing pathogens and tumor cells by producing large amounts of proinflammatory cytokines and expressing MHC molecules [27]. Moreover, the higher proportion of Tregs was found at HCC patients with lower C2 expression in the ICGC cohort, which may also contribute to the unfavorable prognosis of HCC patients as Treg cells are immunosuppressive cells and could promote the occurrence and development of HCC by inhibiting the function of $\mathrm{T}$ cells [28]. Taken together, the interaction of C2 with tumor-infiltrating immune cells may influence the prognosis of HCC.

In addition, to analyze the association of $\mathrm{C} 2$ expression with tumor-infiltrating immune cells, we also explore the underlying signaling pathways exploited by C2 to influence prognosis by KEGG analysis. Signaling pathways, such as "AMPK signaling pathway" and "PPAR signaling pathway," were found to be significantly enriched in HCC patients with higher C2 expression from all three HCC cohorts, which have been reported to take part in the development and progression of HCC. For example, Han et al. have observed that hispidulin could suppress the growth and metastasis of HCC through AMPK signaling-mediated PPAR $\gamma$ activation both in vitro and in vivo [29]. Tuo et al. have also found that phosphoenolpyruvate carboxykinase 1 (PCK1), one of the key enzymes of gluconeogenesis, could inhibit the progression of cell cycle and proliferation of hepatoma cells via the AMPK/p $27^{\mathrm{Kip} 1}$ axis [30]. Basing on these studies, we speculated that $\mathrm{C} 2$ may influence the prognosis of HCC by regulation of the AMPK signaling pathway and/or PPAR signaling pathway.

Several limitations of the present study should also be noted. First, complement is mainly synthesized in the liver and then secreted into blood, but we do not analyze the difference of serum $\mathrm{C} 2$ between $\mathrm{HCC}$ and healthy controls and the prognostic value of serum C2 in HCC. Second, C2 expression is found to be associated with tumor-infiltrating cells, such as CD4 T cells and macrophage M0 cells, but we do not explore how $\mathrm{C} 2$ regulate these immune cells to influence the prognosis of HCC patients. Finally, we do not validate the KEGG pathways enriched in HCC patients with higher $\mathrm{C} 2$ expression in in vitro studies.

In conclusion, higher $\mathrm{C} 2$ expression is associated with better prognosis of HCC, and C2 may influence the prognosis of HCC by interaction with CD4 T cells and macrophage M0 cells and regulation of pathways, such as the AMPK signaling pathway and PPAR signaling pathway.

\section{Abbreviations}

HCC: Hepatocellular carcinoma

TME: Tumor microenvironment

C7: $\quad$ Complement component 7

CFH: Complement factor $\mathrm{H}$

PDT: Photodynamic therapy
C5a: Complement component 5a

C2: $\quad$ Complement component 2

SNP: Single nucleotide polymorphism

TCGA: The Cancer Genome Atlas

GEO: Gene Expression Omnibus

ICGC: International Cancer Genome Consortium

OS: $\quad$ Overall survival

KEGG: Kyoto Encyclopedia of Genes and Genomes

DAVID: Database for Annotation, Visualization, and Integrated Discovery

DEGs: Differentially expressed genes

NSCLC: Non-small-cell lung cancer

MDSCs: Myeloid-derived suppressor cells

EMT: Epithelial-mesenchymal transition

MBL: Mannan binding lectin

PCK1: Phosphoenolpyruvate carboxykinase 1.

\section{Data Availability}

All the data used in the present study could be downloaded from TCGA (https://cancergenome.nih.gov/), GEO (https:// www.ncbi.nlm.nih.gov/geo/), and ICGC portal (https://dcc .icgc.org/projects/LIRI-JP).

\section{Conflicts of Interest}

The authors declare that they have no conflicts of interest.

\section{Authors' Contributions}

Gang Ning, Yan-Lin Huang, and Li-Min Zhen have contributed equally to this work.

\section{Acknowledgments}

This study was supported by grants from the Natural Science Foundation of China (Nos. 81472259, 81570539, and 81873572), National Major Science and Technology Project for the Prevention and Treatment of AIDS and Viral Hepatitis (2018ZX10302205-002 and 2018ZX10302204), Natural Science Foundation of Guangdong Province (2014A030313042), Guangzhou Science and Technology Plan Project (201904010442), Guangdong Provincial Science and Technology Projects (2015A020212007), Sun Yat-sen University Clinical Research 5010 Program (2018009), Research Project on Degree and Postgraduate Education Reform in Guangdong Province (2018JGXM04), Young Teacher Training Program of Sun Yat-sen University (16ykpy40), Open Project of Key Lab of Tropical Disease Control (Sun Yat-sen University), Ministry of Education (2019kfkt07), and Ten Five-Year Plan Fund of the Third Affiliated Hospital of Sun Yat-sen University (SW201701).

\section{References}

[1] R. L. Siegel, K. D. Miller, and A. Jemal, "Cancer statistics, 2019," CA: a cancer journal for clinicians, vol. 69, no. 1, pp. 7-34, 2018.

[2] P. Tabrizian, G. Jibara, B. Shrager, M. Schwartz, and S. Roayaie, "Recurrence of hepatocellular cancer after 
resection: patterns, treatments, and prognosis," Annals of surgery., vol. 261, no. 5, pp. 947-955, 2015.

[3] D. F. Quail and J. A. Joyce, "Microenvironmental regulation of tumor progression and metastasis," Nature medicine., vol. 19, no. 11, pp. 1423-1437, 2013.

[4] D. Hanahan and L. M. Coussens, "Accessories to the crime: functions of cells recruited to the tumor microenvironment," Cancer Cell, vol. 21, no. 3, pp. 309-322, 2012.

[5] M. V. Novikova, N. V. Khromova, and P. B. Kopnin, "Components of the hepatocellular carcinoma microenvironment and their role in tumor progression," Biochemistry Biokhimiia., vol. 82, no. 8, pp. 861-873, 2017.

[6] D. Moris, E. W. Beal, J. Chakedis et al., "Role of exosomes in treatment of hepatocellular carcinoma," Surgical oncology., vol. 26, no. 3, pp. 219-228, 2017.

[7] D. Ricklin, G. Hajishengallis, K. Yang, and J. D. Lambris, "Complement: a key system for immune surveillance and homeostasis," Nature Immunology, vol. 11, no. 9, pp. 785797, 2010.

[8] E. S. Reis, D. C. Mastellos, D. Ricklin, A. Mantovani, and J. D. Lambris, "Complement in cancer: untangling an intricate relationship," Nature reviews Immunology., vol. 18, no. 1, pp. 5$18,2018$.

[9] H. S. Seol, S. E. Lee, J. S. Song et al., "Complement proteins C7 and CFH control the stemness of liver cancer cells via LSF-1," Cancer letters., vol. 372, no. 1, pp. 24-35, 2016.

[10] F. Li, Y. Cheng, J. Lu, R. Hu, Q. Wan, and H. Feng, "Photodynamic therapy boosts anti-glioma immunity in mice: a dependence on the activities of T cells and complement C3," Journal of Cellular Biochemistry, vol. 112, no. 10, pp. 3035-3043, 2011.

[11] L. Gunn, C. Ding, M. Liu et al., "Opposing roles for complement component $\mathrm{C} 5 \mathrm{a}$ in tumor progression and the tumor microenvironment," Journal of Immunology, vol. 189, no. 6, pp. 2985-2994, 2012.

[12] S. Namgoong, J. G. Shin, H. S. Cheong et al., "Genetic association of complement component 2 variants with chronic hepatitis B in a Korean population," Liver international: official journal of the International Association for the Study of the Liver., vol. 38, no. 9, pp. 1576-1582, 2018.

[13] R. J. Clifford, J. Zhang, D. M. Meerzaman et al., "Genetic variations at loci involved in the immune response are risk factors for hepatocellular carcinoma," Hepatology, vol. 52, no. 6, pp. 2034-2043, 2010.

[14] D. H. Parks, G. W. Tyson, P. Hugenholtz, and R. G. Beiko, "STAMP: statistical analysis of taxonomic and functional profiles," Bioinformatics, vol. 30, no. 21, pp. 3123-3124, 2014.

[15] Z. Yin, C. Dong, K. Jiang et al., "Heterogeneity of cancerassociated fibroblasts and roles in the progression, prognosis, and therapy of hepatocellular carcinoma," Journal of hematology \& oncology., vol. 12, no. 1, p. 101, 2019.

[16] T. Imamura, M. Yamamoto-Ibusuki, A. Sueta et al., "Influence of the C5a-C5a receptor system on breast cancer progression and patient prognosis," Breast Cancer, vol. 23, no. 6, pp. 876-885, 2016.

[17] K. Lin, S. He, L. He et al., "Complement component 3 is a prognostic factor of nonsmall cell lung cancer," Molecular medicine reports., vol. 10, no. 2, pp. 811-817, 2014.

[18] R. J. Tesi, "MDSC; the most important cell you have never heard of," Trends in pharmacological sciences., vol. 40, no. 1, pp. 4-7, 2019.
[19] L. Corrales, D. Ajona, S. Rafail et al., "Anaphylatoxin C5a creates a favorable microenvironment for lung cancer progression," Journal of Immunology, vol. 189, no. 9, pp. 4674-4683, 2012.

[20] M. Garnelo, A. Tan, Z. Her et al., "Interaction between tumour-infiltrating B cells and T cells controls the progression of hepatocellular carcinoma," Gut, vol. 66, no. 2, pp. 342-351, 2017.

[21] N. Imai, I. Tawara, M. Yamane, D. Muraoka, H. Shiku, and H. Ikeda, " $\mathrm{CD}^{4+} \mathrm{T}$ cells support polyfunctionality of cytotoxic $\mathrm{CD}^{+} \mathrm{T}$ cells with memory potential in immunological control of tumor," Cancer Science, 2020.

[22] J. Fu, Z. Zhang, L. Zhou et al., "Impairment of $\mathrm{CD} 4^{+}$cytotoxic $\mathrm{T}$ cells predicts poor survival and high recurrence rates in patients with hepatocellular carcinoma," Hepatology, vol. 58, no. 1, pp. 139-149, 2013.

[23] Y.-W. Hsiao, L.-T. Chiu, C.-H. Chen, W.-L. Shih, and T.-P. Lu, "Tumor-infiltrating leukocyte composition and prognostic power in hepatitis B- and hepatitis C-related hepatocellular carcinomas," Genes, vol. 10, no. 8, p. 630, 2019.

[24] R. Ostuni, F. Kratochvill, P. J. Murray, and G. Natoli, “Macrophages and cancer: from mechanisms to therapeutic implications," Trends in immunology., vol. 36, no. 4, pp. 229-239, 2015.

[25] Z. Tian, X. Hou, W. Liu, Z. Han, and L. Wei, "Macrophages and hepatocellular carcinoma," Cell \& Bioscience, vol. 9, no. 1, 2019.

[26] O. W. H. Yeung, C.-M. Lo, C.-C. Ling et al., "Alternatively activated (M2) macrophages promote tumour growth and invasiveness in hepatocellular carcinoma," Journal of hepatology, vol. 62, no. 3, pp. 607-616, 2015.

[27] C. Lamagna, M. Aurrand-Lions, and B. A. Imhof, "Dual role of macrophages in tumor growth and angiogenesis," Journal of leukocyte biology., vol. 80, no. 4, pp. 705-713, 2006.

[28] H. Q. Zhao, W. M. Li, Z. Q. Lu, and Y. M. Yao, "Roles of Tregs in development of hepatocellular carcinoma: a meta-analysis," World journal of gastroenterology., vol. 20, no. 24, pp. 79717978, 2014.

[29] M. Han, H. Gao, P. Ju et al., "Hispidulin inhibits hepatocellular carcinoma growth and metastasis through AMPK and ERK signaling mediated activation of PPAR $\gamma$," Biomedicine \& Pharmacotherapy, vol. 103, pp. 272-283, 2018.

[30] L. Tuo, J. Xiang, X. Pan et al., "PCK1 negatively regulates cell cycle progression and hepatoma cell proliferation via the AMPK/p27 $7^{\text {Kip1 }}$ axis," Journal of experimental \& clinical cancer research: CR., vol. 38, no. 1, p. 50, 2019. 